\title{
Enhancement of electron spin coherence by optical preparation of nuclear spins
}

\author{
Dimitrije Stepanenko and Guido Burkard \\ Department of Physics and Astronomy, University of Basel, \\ Klingelbergstrasse 82, CH-4056 Basel, Switzerland \\ Geza Giedke and Atac Imamoglu \\ Institute of Quantum Electronics, ETH Zürich, CH-8093 Zürich, Switzerland
}

\begin{abstract}
We study a large ensemble of nuclear spins interacting with a single electron spin in a quantum dot under optical excitation and photon detection. When a pair of applied laser fields satisfy two-photon resonance between the two ground electronic spin states, detection of light scattering from the intermediate exciton state acts as a weak quantum measurement of the effective magnetic (Overhauser) field due to the nuclear spins. If the spin were driven into a coherent population trapping state where no light scattering takes place, then the nuclear state would be projected into an eigenstate of the Overhauser field operator and electron decoherence due to nuclear spins would be suppressed: we show that this limit can be approached by adapting the laser frequencies when a photon is detected. We use a Lindblad equation to describe the time evolution of the driven system under photon emission and detection. Numerically, we find an increase of the electron coherence time from $5 \mathrm{~ns}$ to $500 \mathrm{~ns}$ after a preparation time of 10 microseconds.
\end{abstract}

Introduction. Single electron spins localized in small artificial structures, such as semiconductor quantum dots (QDs), have become available and to a large extent controllable [1, 2, 3, 4]. Of particular interest is the phase coherence of such electron spins as single quantum objects, both from a fundamental physics point of view and because of their potential use as quantum bits (qubits) for quantum information processing [6, 7].

A number of physical mechanisms that lead to the gradual reduction of the quantum phase coherence (decoherence) of the electron spin have been analyzed [8]. It has been established experimentally and theoretically that for an electron in a GaAs QD, the predominant decoherence mechanism is the hyperfine coupling to the nuclear spins in the host material 9, 10, 11, 12, 13, 14]. For an unpolarized ensemble of $N$ nuclei and an effective hyperfine interaction energy $A$, the dephasing time in the presence of a weak magnetic field is $T_{2}^{*} \sim 1 / \sigma \sim \sqrt{N} / A$ where $\sigma$ is the width of the distribution of nuclear field values $h_{z}$ parallel to the field. In a typical GaAs QD with $A \sim 90 \mu \mathrm{eV}$ or $A / g \mu_{B}=3.5 \mathrm{~T}$ 15, the number of Ga and As nuclei (spin $I=3 / 2$ ) is $N \sim 5 \cdot 10^{5}$ and $T_{2}^{*} \sim 5 \mathrm{~ns}$; this value is supported by the experimental evidence [4, 5]. The $T_{2}^{*}$ decay originates from nuclear ensemble averaging and can be prolonged by narrowing the nuclear spin distribution 13. Another strategy is to polarize the nuclear spins 9 , but this requires a polarization close to $100 \%$ which is currently not available [13]. Two schemes have been proposed to achieve a narrowing of the nuclear spin distribution, based on electron transport 16] and gate-controlled electronic Rabi oscillations 17.

Here, we analyze an optical scheme for nuclear spin preparation that makes use of spin-flip two-photon (Raman) resonance in a driven three-level system (TLS), in analogy to electromagnetically induced transparency (EIT) in atomic systems [18]. The lowest electronic states in a QD formed in a III-V semiconductor (e.g., GaAs)

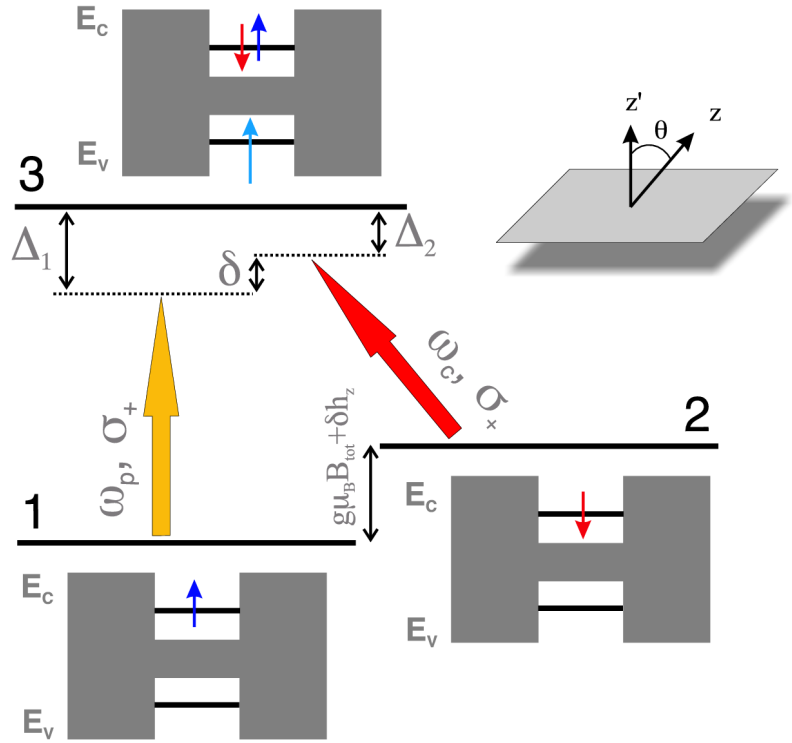

FIG. 1: Three-level system. State 1 (2) is a spin-up (down) conduction-band $\left(E_{C}\right)$ electron, with splitting $g \mu_{B} B_{\text {tot }}+\delta h_{z}$ where $\delta h_{z}$ is the $z$-component of the nuclear (Overhauser) field fluctuations. State 3 is a trion with $J_{z^{\prime}}=3 / 2$. Two laser fields with frequencies $\omega_{p}$ and $\omega_{c}$ are applied near the 13 and 23 resonances with detunings $\Delta_{1,2}$. For $\sigma_{+}$circularly polarized excitation (along $z^{\prime}$ ), both transitions are allowed for $\theta \neq 0$ and transitions to the $J_{z^{\prime}}=-3 / 2$ states are forbidden. Inset: Structural axis $z^{\prime}$, leading to a splitting in $E_{V}$ and spin quantization axis $z \| \mathbf{B}_{\text {tot }}$ in $E_{C}$ where $\cos \theta=z \cdot z^{\prime}<1$.

that are optically active under $\sigma_{+}$circularly polarized excitation are a Zeeman-split single electron in the localized conduction band $\left(E_{C}\right)$ ground state and the negatively charged exciton (trion) $|X\rangle$, i.e., two electrons (spin up and down) plus one valence band heavy hole (hh) with angular momentum $J_{z^{\prime}}=+3 / 2$ (Fig. 1). The 
$J=3 / 2$ sector in the valence band is split into lh and hh states along the quantization axis $z^{\prime}$ determined by the direction of strong QD confinement. Here, we assume excitation from the hh $\left(J_{z^{\prime}}= \pm 3 / 2\right)$ subband only. The quantization axis $z$ in $E_{C}$ is parallel to the total magnetic field $\mathbf{B}_{\text {tot }}$, and we assume that the axes $z$ and $z^{\prime}$ enclose an angle $\theta>0$. The spin up and down states in $E_{C}$ are then $|\uparrow\rangle \equiv|\uparrow\rangle_{z}=\cos (\theta)|\uparrow\rangle_{z^{\prime}}+\sin (\theta)|\downarrow\rangle_{z^{\prime}}$ and $|\downarrow\rangle \equiv \mid \downarrow$ \rangle$_{z}=\cos (\theta)|\downarrow\rangle_{z^{\prime}}-\sin (\theta)|\uparrow\rangle_{z^{\prime}}$. From now on, we drop the index $z$. Two circularly polarized $\left(\sigma_{+}\right)$continuouswave lasers at the frequencies $\omega_{p}=\omega_{X}-\omega_{\uparrow}-\Delta_{1}$ and $\omega_{c}=\omega_{X}-\omega_{\downarrow}-\Delta_{2}$ stimulate the transitions between $|\uparrow\rangle$ and $|X\rangle$ and between $|\downarrow\rangle$ and $|X\rangle$, while the trion with $J_{z^{\prime}}=-3 / 2$ is not excited.

The mechanism leading to a narrowing of the nuclear field distribution $\nu$ works as follows. The population of the excited state $|X\rangle$, and thus the probability of photon emission (scattering) is only non-zero away from the twophoton resonance $\delta=0$ where $\delta=\Delta_{1}-\Delta_{2}$. In the presence of the nuclear spins this resonance moves to $\delta=$ $\delta h_{z}$ where $\delta h_{z}$ is the deviation of the nuclear field (along $z$ ) from its mean $\left\langle h_{z}\right\rangle$. The absence of photon emission during a waiting time $t$ constitutes a weak measurement of the quantum operator $\delta h_{z}$. In the limit $t \rightarrow \infty$, it becomes a strong measurement, projecting the nuclear state onto $\left|\delta h_{z}=0\right\rangle$ (width $\sigma=0$ ), thus eliminating electron decoherence due to the fluctuating field $\delta h_{z}$.

Model. The Hamiltonian for the TLS coupled to nuclei,

$$
H=H_{0}+H_{\mathrm{int}}+H_{\mathrm{hf}},
$$

contains the three energy levels in $H_{0}=-\frac{\hbar \omega_{z}}{2} \Sigma_{z}+$ $\hbar \omega_{X} P_{X}$, with $\Sigma_{i}=\left(\begin{array}{cc}\sigma_{i} & 0 \\ 0 & 0\end{array}\right)$ and $P_{X}=|X\rangle\langle X|=$ $(001)^{T}(001)$. The spin splitting is given as $\hbar \omega_{z}=$ $g \mu_{B} B_{\text {tot }}=\left|g \mu_{B} \mathbf{B}+\langle\mathbf{h}\rangle\right|$, the sum of the external magnetic and the mean nuclear fields. The nuclear (Overhauser) field operator is $\mathbf{h}=\sum_{i=1}^{N} A_{i} \mathbf{I}_{i}$, where $A_{i}=$ $a_{i} v_{0}\left|\Psi\left(\mathbf{r}_{i}\right)\right|^{2}$, and $\Psi\left(\mathbf{r}_{i}\right)$ denotes the electron wave function at the position $\mathbf{r}_{i}$ of the $i$ th atomic nucleus and $v_{0}$ is the volume of the unit cell. The classical laser fields in the rotating wave approximation (RWA) are described by [18] $H_{\text {int }}=\Omega_{p} e^{i \omega_{p} t}|X\rangle\left\langle\uparrow\left|+\Omega_{c} e^{i \omega_{c} t}\right| X\right\rangle\langle\downarrow|+$ h.c. The coupling of the electron spin to the quantum fluctuations of the Overhauser field $\mathbf{h}$, will be described by the term $H_{\mathrm{hf}}=-\frac{1}{2} \delta \mathbf{h} \cdot \mathbf{\Sigma}$, where $\delta \mathbf{h}=\mathbf{h}-\langle\mathbf{h}\rangle$. In the rotating frame $\tilde{\Psi}(t)=U(t) \Psi(t)$ with $U(t)=e^{-i \omega_{p} t} P_{\uparrow}+$ $e^{-i \omega_{c} t} P_{\downarrow}+P_{X}$, where $P_{\uparrow}=|\uparrow\rangle\langle\uparrow|$ and $P_{\downarrow}=|\downarrow\rangle\langle\downarrow|$, we find $\tilde{H}(t)=U(t)\left[H(t)+\hbar \omega_{p} P_{\uparrow}+\hbar \omega_{c} P_{\downarrow}\right] U(t)^{\dagger}$, and, up to constant terms proportional to the unity matrix (we drop the tilde and use $H$ for the Hamiltonian henceforth),

$$
H(t)=-\frac{\hbar}{2}\left(\begin{array}{ccc}
\delta & 0 & \Omega_{p} \\
0 & -\delta & \Omega_{c} \\
\Omega_{p} & \Omega_{c} & -\Delta
\end{array}\right)-\frac{\hbar}{2} \delta h_{z} \Sigma_{z}+H_{\perp}
$$

where $\Delta=\Delta_{1}+\Delta_{2}$. The hyperfine flip-flop terms $H_{\perp}=$ $\hbar\left(\delta h_{+} \Sigma_{-} e^{i t\left(\omega_{p}-\omega_{c}\right)}+\delta h_{-} \Sigma_{+} e^{-i t\left(\omega_{p}-\omega_{c}\right)}\right) / 4$ are oscillating rapidly at the frequency $\omega_{p}-\omega_{c}=g \mu_{B} B_{\text {tot }} / \hbar-\delta$ and can be neglected in the RWA [19], leading to a blockdiagonal Hamiltonian $H=\operatorname{diag}\left(H_{1}, H_{2}, \ldots, H_{K}\right)$, with

$$
H_{k}=-\frac{\hbar}{2}\left(\begin{array}{ccc}
\delta h_{z}^{k}+\delta & 0 & \Omega_{p} \\
0 & -\delta h_{z}^{k}-\delta & \Omega_{c} \\
\Omega_{p} & \Omega_{c} & -\Delta
\end{array}\right),
$$

where $\delta h_{z}^{k}$ are the eigenvalues of the operator $\delta h_{z}$. The state of the TLS combined with the nuclear spins is described by the density matrix $\rho$, which we divide up into 3 -by-3 blocks $\rho_{k k^{\prime}}$. The density matrix evolves according to the generalized master equation [18]

$$
\dot{\rho}=\mathcal{L} \rho \equiv \frac{1}{i \hbar}[H, \rho]+\mathcal{W} \rho,
$$

with the Hamiltonian Eq. (2) and the dissipative term $\mathcal{W} \rho=\sum_{\alpha=\uparrow, \downarrow} \Gamma_{X \alpha}\left(2 \sigma_{\alpha X} \rho \sigma_{X \alpha}-\sigma_{X X} \rho-\rho \sigma_{X X}\right) / 2+$ $\sum_{\beta=\downarrow, X} \gamma_{\beta}\left(2 \sigma_{\beta \beta} \rho \sigma_{\beta \beta}-\sigma_{\beta \beta} \rho-\rho \sigma_{\beta \beta}\right) / 2$, where $\sigma_{i j}=$ $\sigma_{i j} \otimes \mathbb{1}=|i\rangle\langle j|$ acts on the TLS only. The rate $\Gamma_{X \alpha}$ describes the radiative decay of the exciton $|X\rangle$ into one of the single-electron states $\alpha=|\uparrow\rangle,|\downarrow\rangle$, while $\gamma_{\beta}$ is the pure dephasing rate of state $\beta=|\downarrow\rangle,|X\rangle$ with respect to $|\uparrow\rangle$. Since $H$ has block form, the master equations for the various blocks are decoupled, and have the closed form

$$
\dot{\rho}_{k k^{\prime}}=\frac{1}{i \hbar}\left(H_{k} \rho_{k k^{\prime}}-\rho_{k k^{\prime}} H_{k^{\prime}}\right)+\mathcal{W} \rho_{k k^{\prime}} .
$$

The diagonal blocks obey the familiar Lindblad equation,

$$
\dot{\rho}_{k k}=\mathcal{L}_{k} \rho_{k k}, \quad \mathcal{L}_{k}=-i\left[H_{k}, \rho\right]+\mathcal{W} \rho .
$$

Stationary state. We start with the factorized initial state $\rho_{0}=\chi_{0} \otimes \nu_{0}$ with arbitrary initial density matrices $\chi_{0}$ and $\nu_{0}$ of the TLS and the nuclear ensemble, where $\nu_{0}=\sum_{k k^{\prime}} \nu_{k k^{\prime}}\left|\delta h_{z}^{k}\right\rangle\left\langle\delta h_{z}^{k^{\prime}}\right|$, and $\left|\delta h_{z}^{k}\right\rangle$ are eigenstates of the Overhauser operator, $\delta h_{z}\left|\delta h_{z}^{k}\right\rangle=\delta h_{z}^{k}\left|\delta h_{z}^{k}\right\rangle$. As the off-diagonal elements of $\nu_{0}$ turn out to be irrelevant for the stationary state of the TLS, our analysis is valid both for a pure initial state (e.g., with $\nu_{k k^{\prime}}=\sqrt{\nu_{k k} \nu_{k^{\prime} k^{\prime}}}$ ) and for a mixed initial state (e.g., the completely mixed state, $\left.\nu_{k k^{\prime}} \propto \delta_{k k^{\prime}}\right)$. We assume a Gaussian distribution $\nu_{k k}=$ $(2 \pi)^{-1 / 2} \sigma^{-1} \exp \left[-\left(\delta h_{z}^{k}\right)^{2} / 2 \sigma^{2}\right]$, with an initial width $\sigma=$ $\sigma_{0}=A / \sqrt{N}$, plotted as a solid line in Fig. 2(a). For our numerical calculations we choose $A=90 \mu \mathrm{eV}$ and $N \approx 5 \cdot 10^{5}$ corresponding to $\sigma_{0} \simeq 0.13 \mu \mathrm{eV} \simeq 0.2 \hbar \Gamma$ with $\Gamma=1 \mathrm{~ns}$. We choose a sample of $n \ll(2 I+1)^{N}$ states from the total Hilbert space $(n \sim 4000)$.

Due to the hyperfine coupling between the TLS and the nuclei, the two systems become entangled as the stationary state is reached. The stationary density matrix $\bar{\rho}$ is independent of $\chi_{0}$ and has the general form $\bar{\rho}=\sum_{k k^{\prime}} \bar{\rho}_{k k^{\prime}} \otimes\left|\delta h_{z}^{k}\right\rangle\left\langle\delta h_{z}^{k^{\prime}}\right|$. We derived an analytical expression for the 3 -by-3 diagonal blocks $\bar{\rho}_{k k}$ of $\bar{\rho}$ as a function of all parameters, including $\delta h_{k}$. We find numerically that the off-diagonal blocks $\rho_{k k^{\prime}}\left(k \neq k^{\prime}\right)$, i.e., the nuclear coherences, decay exponentially and thus vanish in the stationary density matrix $\bar{\rho}$. Therefore, $\bar{\rho}$ is blockdiagonal, $\bar{\rho}_{k k^{\prime}}=\delta_{k k^{\prime}} \bar{\rho}_{k k}$. 


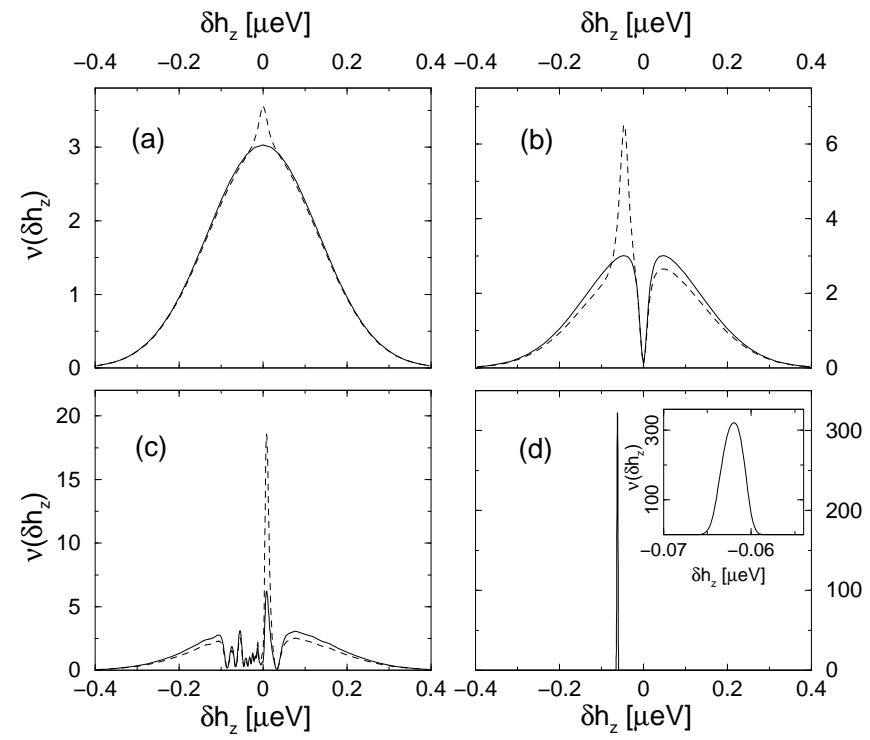

FIG. 2: Conditional evolution of the nuclear spin distribution $\nu\left(\delta h_{z}^{k}\right)=\nu_{k k}$. (a) During the first period $t_{1}$ of evolution without photon emission, the initial Gaussian distribution (solid line) develops a peak at the two-photon resonance (dashed line). (b) Change of $\nu\left(\delta h_{z}\right)$ after emission at $t_{1}$ (solid line), until before emission time $t_{2}$ of the second photon (dashed line). The two-photon resonance $\delta$ has been shifted to the position of the left maximum (adaptive technique). The depleted region around $\delta h_{z}^{k}=0$ is developed at $t_{1}$. (c) Analogous situation between $t_{11}$ and $t_{12}$. (d) $\nu\left(\delta h_{z}\right)$ is obtained after a total time of $10 \mu \mathrm{s}$. Inset: Magnification of peak in $(\mathrm{d})$. The width of $\nu\left(\delta h_{z}\right)$ is reduced by a factor of $\approx 100$ compared to the initial width in (a). The parameters used for this calculation are $\Omega_{c}=\Omega_{p}=0.2 \mathrm{~ns}^{-1}, \Delta=0, \Gamma_{X \uparrow}=\Gamma_{X \downarrow}=1 \mathrm{~ns}^{-1}$, and $\gamma_{\downarrow}=\gamma_{X}=0.001 \mathrm{~ns}^{-1}$.

Evolution of the observed system. In order to enhance the electron spin coherence, we aim at narrowing the nuclear spin distribution. In the case of a Gaussian distribution, this amounts to decreasing the width $\sigma$ with respect to its initial value $\sigma_{0}$, thus increasing the electron coherence time $t_{0} \simeq 1 / 2 \sigma$. Ideally, we would perform a projective measurement $P$ on the nuclear spins such that $P \bar{\rho}_{k k} P \propto \delta\left(\delta h_{z}^{k}-\delta\right)$. This also bounds the off-diagonal elements of $\nu=\operatorname{Tr}_{\Lambda} \rho$, where $\operatorname{Tr}_{\Lambda}$ is the partial trace over the TLS, because $\left|\nu_{k k^{\prime}}\right| \leq \sqrt{\nu_{k k} \nu_{k^{\prime} k^{\prime}}}$ due to the positivity of $\nu$. The measurement $P$ can be successively approximated by monitoring the photon emission from the QD. The longer the period $t$ during which no photon is emitted, the higher is the probability for $\delta h_{z}$ to be at the two-photon resonance, $\delta h_{z}=\delta$.

To describe the state of the system conditional on a certain measurement record, we use the conditional density matrix $\rho^{c}$. In the absence of photon emission, $\rho_{c}$ obeys Eq. (6) with $\mathcal{L}_{k}$ replaced by $\mathcal{L}_{k}-\mathcal{S}$, with the collapse operator $\mathcal{S}$ [20],

$$
\dot{\rho}_{k k}^{c}=\left(\mathcal{L}_{k}-\mathcal{S}\right) \rho_{k k}^{c}, \quad \mathcal{S} \rho=\sum_{\alpha=\uparrow, \downarrow} \Gamma_{X \alpha} \sigma_{\alpha X} \rho \sigma_{X \alpha} .
$$

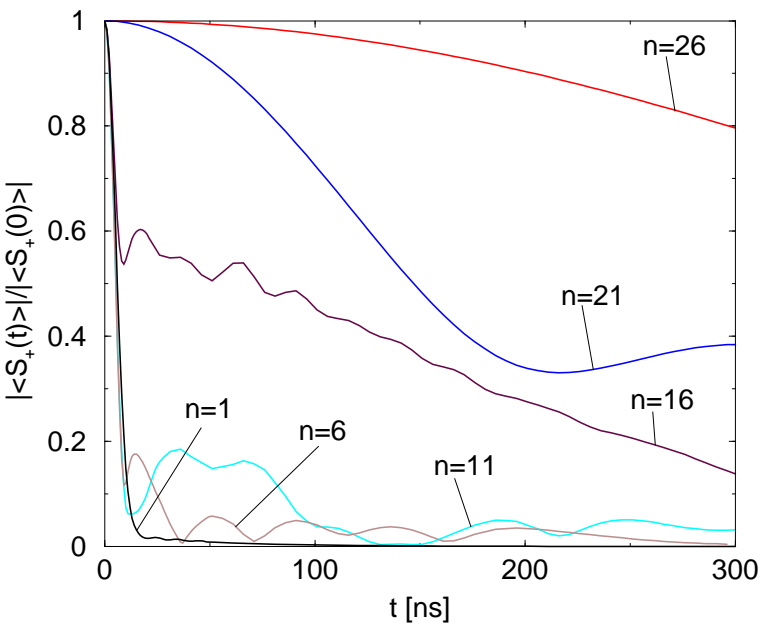

FIG. 3: Electron coherence function $\left|\left\langle S_{+}(t)\right\rangle\right| /\left|\left\langle S_{+}(0)\right\rangle\right|$ vs. electronic precession time $t$ calculated from $\nu\left(\delta h_{z}\right)$ in Fig. 2 after emission of the $n$th photon $(n=1,6, \ldots, 26)$. The initial decay is approximately Gaussian.

We have numerically calculated $\rho^{c}$ conditional on the absence of emitted photons for a given duration $t$. We plot the updated probability distribution $\nu_{k k}$ from $\nu=\operatorname{Tr}_{\Lambda} \rho^{c}$ as a dashed line in Fig.2(a). We find that the a posteriori probability is concentrated around the two-photon resonance. Of course, this process will eventually be stopped by the emission of a photon.

Photon emission. The stationary emission rate is 20.

$$
\Gamma_{\mathrm{em}}=\operatorname{Tr} \mathcal{S} \bar{\rho}(t)=\Gamma \sum_{k}\left(\rho_{k k}\right)_{X X} \nu_{k k},
$$

where $\Gamma=\Gamma_{X \uparrow}+\Gamma_{X \downarrow}$. The average number of photons emitted during time $t$ is $\left\langle N_{\mathrm{ph}}\right\rangle=t \Gamma_{\mathrm{em}}$ and the a priori probability for $N_{\mathrm{ph}}=0$ is, according to Poissonian statistics, $P_{\text {dark }}(t)=\exp \left(-\Gamma_{\mathrm{em}} t\right)$. The waiting time distribution for photon emissions is $p_{\text {wait }}(t)=\Gamma_{\mathrm{em}}^{-1} \exp \left(-\Gamma_{\mathrm{em}} t\right)$ with a mean waiting time $\langle t\rangle=\Gamma_{\mathrm{em}}^{-1}$. The progressive narrowing of the Overhauser field distribution, Eqs. (7) and (8), leads to a decreasing photon emission rate $\Gamma_{\mathrm{em}}$, and therefore to an increasing average waiting time $t$. We first assume that every emitted photon is detected and later generalize to the case of imperfect detection.

Using Eq. (8), we find for the update rule of the nuclear density matrix upon photon emission $\nu^{\prime}=$ $\operatorname{Tr}_{\Lambda} \mathcal{S} \rho^{c} / \operatorname{Tr} \mathcal{S} \rho^{c}$. The Overhauser field distribution after the emission is

$$
\nu_{k k}^{\prime}=\frac{\nu_{k k}\left(\rho_{k k}\right)_{X X}}{\sum_{j} \nu_{j j}\left(\rho_{j j}\right)_{X X}},
$$

where $\nu_{k k}$ and $\left(\rho_{k k}\right)_{X X}=\left\langle X\left|\rho_{k k}\right| X\right\rangle$ are taken before the emission. According to Eq. (9), the population in the Overhauser field $\delta h_{z}$ corresponding to the two photon resonance $\delta h_{z}=\delta$ is depleted by the photon emission (Fig. 2 b, solid line). However, the combined effect of the observed evolution without emission and the collapse leads to narrowing of the nuclear distribution. 


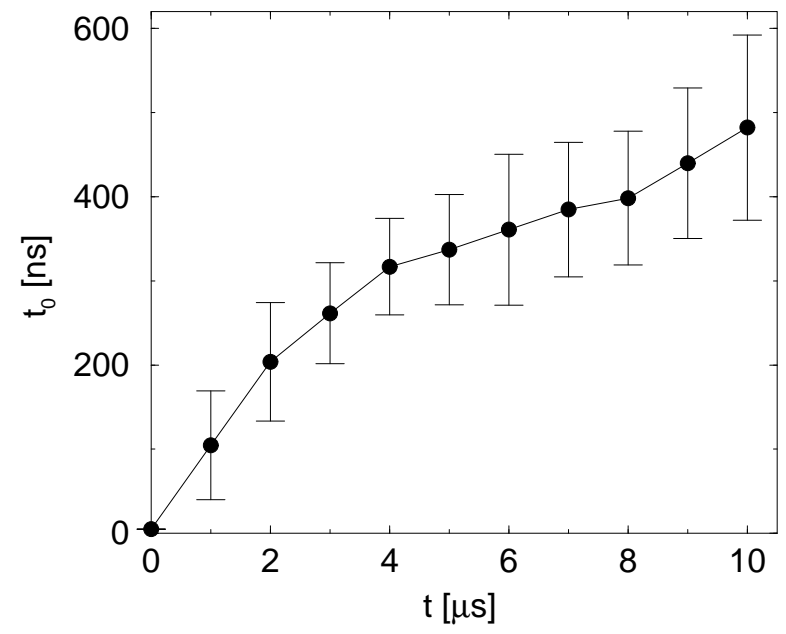

FIG. 4: Characteristic time $t_{0}$ of the initial Gaussian decay in $\left|\left\langle S_{+}(t)\right\rangle\right| /\left|\left\langle S_{+}(0)\right\rangle\right|$ in Fig. [3] as a function of the optical preparation time $t$, averaged over 50 numerical runs (with error bars indicating the standard deviation).

Adaptive technique. The stationary, isolated TLS at the two-photon resonance is in a dark state. However, the coupling to the nuclei introduces a nonzero probability for occupation of the $|X\rangle$ state and for emission of a photon. The detection of a photon provides information about $\delta h_{z}$ and an adjustment of the EIT setup. Thus, photon emission does not necessarily signify a failed attempt to narrow the nuclear field distribution, but can be used as an input for the next weak measurement with adjusted frequencies of the driving lasers, $\omega_{p}^{\prime}=\omega_{p}+\epsilon / 2$ and $\omega_{c}^{\prime}=\omega_{c}-\epsilon / 2$, so that the new two-photon resonance condition is $\delta h_{z}=\delta^{\prime}$ where $\delta^{\prime}=\delta+\epsilon$ while $\Delta^{\prime}=\Delta$. We choose $\epsilon$ such that the new resonance with the Overhauser field lies in one of the two maxima $\delta h_{z}^{\max }$ formed after the photon emission, see Fig. 2b. This situation is formally described by Eq. (3) with the substitution $\delta \rightarrow \delta+\delta h_{z}^{\max }$. We note that the adaptive technique also works by changing only one of the laser frequencies. Right after the photon emission, the TLS is in one of the single electron states, $|\uparrow\rangle$ or $|\downarrow\rangle$. Within a time $1 / \Gamma$, much faster than any nuclear time scale, the driven electronic system will reach the new stationary state. Then, the photon emission from the QD can again be monitored, leading to an enhanced nuclear population at the new resonance (Fig. 2b, dashed line), thus further narrowing the nuclear distribution and further enhancing the electron spin coherence. This procedure can be repeated many times, leading to a nuclear distribution that is limited only by the width of the EIT resonance (Fig. 2],d).

Electron spin decoherence. The electron spin coherence is quantified using the expectation value of the raising operator $S_{+}(t)$ in a state $\left|x_{+}\right\rangle$that is prepared per- pendicular to the total field $\mathbf{B}_{\text {tot }}$ and is freely precessing about the fluctuating nuclear field $\delta h_{z},\left\langle S_{+}(t)\right\rangle \equiv$ $\left\langle x_{+}\left|S_{+}(t)\right| x_{+}\right\rangle=\sum_{k} \nu_{k k}\left\langle\delta h_{z}^{k}\left|\left\langle x_{+}\left|S_{+}(t)\right| x_{+}\right\rangle\right| \delta h_{z}^{k}\right\rangle$. Using $\left\langle\delta h_{z}^{k}\left|S_{+}(t)\right| \delta h_{z}^{k}\right\rangle=e^{i t \delta h_{z}^{k} \sigma_{z} / 2} S_{+}(0) e^{-i t \delta h_{z}^{k} \sigma_{z} / 2}$, we obtain $\left\langle S_{+}(t)\right\rangle=(\hbar / 2) \sum_{k} \nu_{k k} \exp \left(i t \delta h_{z}^{k}\right)$, which we plot in Fig. 3] at various stages in an adaptive optical measurement scheme. We make a Gaussian fit, $\left\langle S_{+}(t)\right\rangle \propto$ $\exp \left(-t^{2} / t_{0}^{2}\right)$, for short times $t$ and plot the coherence time $t_{0}$ as a function of total waiting time in Fig. 4. This is the main result of our theoretical analysis: The repeated observation of the QD photon emission and adaptation of the laser frequencies $\omega_{c}$ and $\omega_{p}$ after each photon emission leads to a pronounced enhancement of the electron coherence time, for the realistic parameters chosen, from $t_{0}=5 \mathrm{~ns}$ to $\approx 500 \mathrm{~ns}$ within a total observation time of $10 \mu \mathrm{s}$.

Imperfect detectors. We cannot expect to have perfect photon detectors at our disposal, therefore we discuss here the case of a detector with efficiency $e<1$. For an imperfect detector, Eq. (7) becomes $\dot{\rho}_{k k}^{c}=\left(\mathcal{L}_{k}-e \mathcal{S}\right) \rho_{k k}^{c}$, reflecting that a photon is only detected with probability $e$. We have numerically analyzed the case of $e=10 \%$ (other parameters as above) and find $t_{0} \approx 460$ ns after a preparation time of $t=50 \mu \mathrm{s}$. The requirement for longer preparation times is a consequence of the reduced photon detection rate $e \Gamma_{\mathrm{em}}$. We note that this is still orders of magnitude shorter than the time after which the nuclear spin diffuses, estimated to be around $0.01 \mathrm{~s}$ due to higherorder hyperfine flip-flop terms [17], but possibly longer due to Knight shift gradient effects. Long time scales for the decay of nuclear polarization, on the order of seconds in the case of nuclear spins in contact with donors in GaAs, have also been seen experimentally [21].

Conclusions. We find that it is possible to efficiently enhance the quantum phase coherence of an electron spin in a QD surrounded by a large ensemble of nuclear spins by a continuous weak measurement of the Overhauser field using optical excitation at a two-photon resonance of the TLS formed by the spin-split conduction band electron and one of the trion states. An intriguing question is whether the electron spin coherence can be enhanced by a quantum Zeno type effect to the point where it is ultimately determined by spin-orbit interaction: since reservoir correlation time of dominant electron spin decoherence due to flip-flop terms of the hyperfine interaction is $\sim 1 \mu \mathrm{s}$, this would most likely require high efficiency detection of the scattered photons.

Acknowledgments. We thank W. A. Coish, D. Klauser, and D. Loss for useful discussions. We acknowledge financial support from the Swiss National Science Foundation (SNF) through an SNF professorship (G.B.) and through NCCR Nanoscience. 
[3] F. H. L. Koppens et al., Science 309, 1346 (2005).

[4] J. R. Petta et al., Science 309, 2180 (2005).

[5] The decay of a spin echo envelope, being a measure for $T_{2} \geq T_{2}^{*}$, can be much slower [4].

[6] D. Loss and D. P. DiVincenzo, Phys. Rev. A 57, 120 (1998).

[7] A. Imamoglu et al., Phys. Rev. Lett. 83, 4204 (1999).

[8] V. Cerletti, W. A. Coish, O. Gywat, and D. Loss, Nanotechnology 16, 27 (2005).

[9] G. Burkard, D. Loss, and D. P. DiVincenzo, Phys. Rev. B 59, 2070 (1999).

[10] A. Khaetskii, D. Loss, and L. Glazman, Phys. Rev. Lett. 88, 186802 (2002).

[11] I. A. Merkulov, A. L. Efros, and M. Rosen, Phys. Rev. B 65, 205309 (2002).

[12] A. Khaetskii, D. Loss, and L. Glazman, Phys. Rev. B 67, 195329 (2003).

[13] W. A. Coish and D. Loss, Phys. Rev. B 70, 195340
(2004).

[14] W. A. Coish and D. Loss, Phys. Rev. B 72, 125337 (2005).

[15] D. Paget, G. Lampel, B. Sapoval, and V. I. Safarov, Phys. Rev. B 15, 5780 (1977).

[16] G. Giedke, J. M. Taylor, D. D'Alessandro, M. D. Lukin, and A. Imamoğlu, quant-ph/0508144

[17] D. Klauser, W. A. Coish, and D. Loss, cond-mat/0510177

[18] M. Fleischhauer, A. Imamoglu, and J. P. Marangos, Rev. Mod. Phys. 77, 633 (2005).

[19] The neglected flip-flop terms contribute to electron-spin decoherence due to higher order terms.

[20] H. J. Carmichael, An Open Systems Approach to Quantum Optics (Springer, Berlin, 1993).

[21] D. Paget, Phys. Rev. B 25, 4444 (1982). 